\title{
ASYMMETRIES IN THE PERCEPTION OF FACIAL AFFECT: IS THERE AN INFLUENCE OF READING HABITS?
}

\author{
JYotsna VAID ${ }_{+}^{*+}$ and Maharaj Singh $\dagger$ \\ *Texas A \& M University, U.S.A.; and †Institute of Advanced Studies, Meerut University, India, 250005
}

(Received 14 October 1987; accepted 10 April 1989)

\begin{abstract}
Perceptions of happy facial affect from asymmetric composite faces presented in free vision were compared in four groups: left-to-right readers (Hindi), right-to-left readers (Arabic), left-to-right and right-to-left readers (Hindi/Urdu) and illiterates (Hindi/Urdu). Right- and left-handed users of Hindi and Urdu were studied. The analysis of asymmetry scores revealed a significant effect of Group, such that a left hemifield preference was present only in the left-to-right (Hindi) group. There were no reliable differences between right- and left-handers. Furthermore, the leftward bias was present in a significantly larger proportion of Hindi than Urdu or A rabic readers. These results are taken to reflect an interaction between a cerebral laterality effect and a directional scanning effect in facial affect judgment.
\end{abstract}

\section{INTRODUCTION}

FINDINGS of functional asymmetries in verbal or nonverbal visual perception tasks are generally interpreted in terms of a hemispheric specialization framework. However, this framework is not the only one available nor the one that was initially considered in early studies of visual hemifield presentation of words. The original interpretation of visual field asymmetries was in terms of post-exposural directional scanning tendencies arising from reading and writing experience $[6,20]$. Visual field asymmetries, in this view, predominantly reflect a tendency to scan information in the direction in which one reads. Thus, a reader of English who is fixating at center will show a right visual ficld (RVF) advantage for unilaterally presented words and a left visual field (LVF) advantage for bilaterally presented words. Indeed, the suggestion has been raised that directional biases arising from reading habits may even generalize to nonverbal material in the visual modality [12] or even to the auditory modality $[2,3]$.

Since the scanning interpretation offers an equally plausible account of perceptual asymmetries as a cerebral laterality interpetation, it is difficult to ascertain the source of the asymmetry if the research is restricted to readers of left-to-right scripts such as English. In view of this problem, a number of studies have considered readers of right-to-left scripts, such as Hebrew [30,35]. Findings from the Hebrew literature are mixed, however, with some studies reporting a right visual field advantage for Hebrew as well as for English and others reporting a weaker RVF advantage or even a LVF advantage in the identification of Hebrew stimuli [37].

¥Address for correspondence: J. Vaid, Department of Psychology, Texas A \& M University, College Station, TX 77843, U.S.A. 


\section{LIMITATIONS OF HEBREW TACHISTOSCOPIC STUDIES}

While suggesting a potentially important role of scanning, the Hebrew tachistoscopic literature does not provide as clear or strong a test of the scanning hypothesis as might be desired. Since these studies either compare Americans with Israelis or consider HebrewEnglish bilinguals, extralinguistic factors such as degree of exposure to each language and context of usage, e.g. the extent to which the written form of each language is integrated into daily life, could bring about differences in performance. Furthermore, linguistic differences between Hebrew and English other than those arising from reading/writing direction, could contribute to the results given the substantial differences between the two languages in phonology, grammar, etc. Even in terms of directionality the two languages are not strictly opposite since many individual letters in Hebrew, especially when written in a non-cursive style, are written from left-to-right, as in English; by contrast, many Arabic letters are written from right-to-left. Right-left directional preferences have in fact been found to be stronger and more consistent for Arabic readers than for Hebrew readers [23]. Finally, in Hebrew, arithmetic and musical notation is written from left to right. Since all children learn arithmetic, even if not all learn musical notation, their exposure to a left-to-right scanning order might further weaken whatever right-to-left scanning biases they might have acquired from reading Hebrew. In view of the above considerations, it is difficult to assess the contribution of the Hebrew tachistoscopic literature to the scanning issue in cerebral laterality research.

\section{THE PRESENT STUDY}

The present study sought to examine the relative contribution of directional scanning biases and cerebral lateralization using a different population of right-to-left readers. Instead of using readers of Hebrew, we studied readers of Arabic and of Urdu, a derivative of Arabic used among Muslims in India and Pakistan. The left-to-right reading group we sludied were readers of Hindi, a language written in the Devanagari script and spoken mainly in North India. Hindi and Urdu have an interesting property: they are practically identical on the spoken level, sharing a common lexicon, phonology and grammar, but they differ radically on the written level [32] see Fig. 1. Of relevance to the issue at hand is the difference in reading and writing direction: single letters and words in Hindi are written and read from left to right while in Urdu they are written and read from right to left.

An additional aim of this study was to explore whether reading scan biases arise in performance on nonlinguistic tasks. This issue is important since perceptual asymmetries on nonlinguistic tasks are typically interpreted solely in terms of a cerebral lateralization framework and specifically in terms of right hemisphere specialization. For example, while reading habit has been acknowledged as a potential factor influencing aesthetic perception, its influence has never been empirically assessed and over time a cerebral laterality interpretation has become the accepted explanation of perceptual asymmetries in aesthetic judgment $[13,26]$. A similar bias exists in the literature on facial affect recognition (see $[7,9$, $16,37]$ for reviews). The consistent finding from these studies is a left visual field advantage, which emerges in a variety of different tasks and conditions, leading LEY and STRAuss [29], to conclude that for most right-handed people, "the right hemisphere plays the critical role in recognizing both positive and negative emotional facial expressions", (p. 278). Such a conclusion may well be warranted but, strictly speaking, it is premature given that the relative influence of another variable, namely, directional biases related to reading habits, 


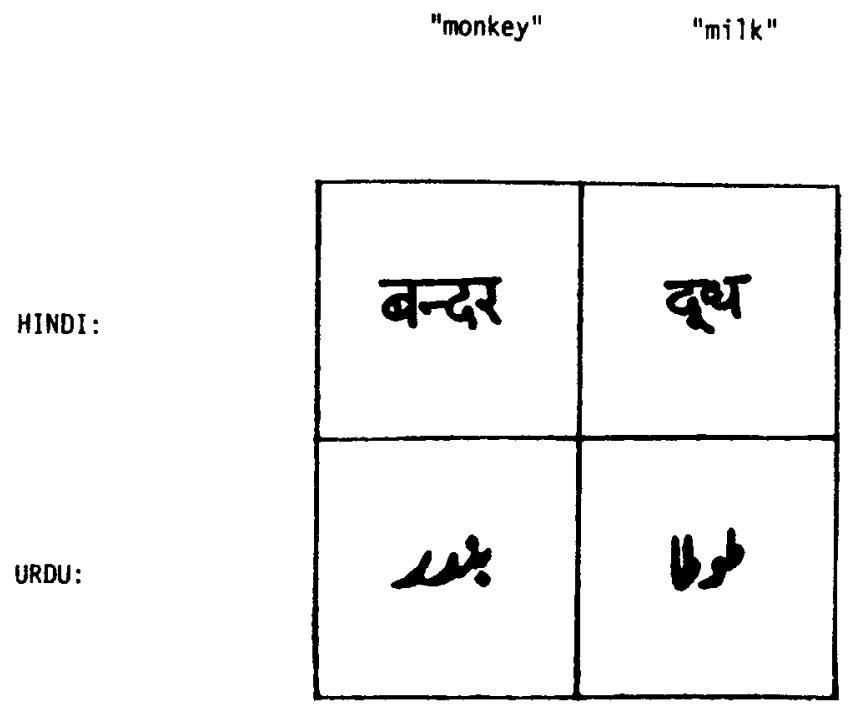

Fig. 1. Examples of two words written in Hindi and in Urdu script.

has never been assessed inasmuch as all the subjects in these studies have been readers of a left-to-right script.

\section{CHIMERIC FACIAL AFFECT JUDGMENT TEST}

One particularly popular procedure used to study facial affect perception is that developed by CAMPBELL [8] and adapted by JERRE LEVY [18]. Subjects are shown pairs of asymmetric chimeric faces in which one half of the poser's face is smiling and the other half is in a neutral pose. For one member of the pair, the smiling half of the face is to the viewer's right; in the other face it is to the left. Subjects are to choose the face that looks happier. Stimuli need not be presented tachistoscopically, for significant lateral preferences are obtained even in a freeviewing situation. The feasibility of this paradigm and its apparent lack of contamination from verbal processing ("the task clearly avoids verbal processing demands" according to one group of researchers, see [19], p. 179) have made it an especially promising noninvasive experimental procedure for assessing hemispheric specialization of emotion in diverse populations. In 10 out of 11 replications using this paradigm $[4,5,8,10,11,18,19,21,25,27$, 33], including one in which infant chimeric faces were viewed by adult subjects [4] a significant left field preference was noted in right-handed adults. That is, subjects chose that face as happier in which the smile appeared to their left. The only exception was a study by NATALE et al. [33], which was also the only study with a slightly different procedure (see [28], p. 281, for further discussion). In Best's study [4], the left field preference was more pronounced for judgments of sad than of happy expressions. Among left-handers, free-vision studies of affect judgment of chimeric faces typically find no significant asymmetry when the sample sizes are small or moderate [18] and a small leftward bias that is significantly smaller than that of right-handers when the sample size is large ([27]; but see [10]). LEVINE and LEVY [25] suggest that the left field bias found in the face perception task "may in part 
reflect an attentional bias toward the left induced by selective activation of the right homisphere which is specialized for face processing" (p. 292).

\section{HYPOTHESES}

If the above biological account of the left field bias in facial affect judgments is the primary explanation, a significant left field preference should be observed regardless of the directionality of the user's script. Individual differences might possibly emerge only between right- and left-handers, inasmuch as handedness differences are traditionally viewed as being rooted in biology. Alternatively, if a cultural, i.e. reading-habit account is a valid explanation of the observed asymmetry, a left field preference should be observed in left-to-right readers and a right field preference should be observed in right-to-left readers. By the same logic, illiterates (i.e. those who have not acquired any reading or writing experience), and bidirectional readers of left-to-right and right-to-left scripts should show no consistent asymmetry on this task. A third possibility is that both a biological and a cultural influence are operating, in which case the size of the left field preference in right-to-left readers should be smaller than that evidenced by left-to-right readers. To investigate these possibilities it is necessary to compare users of languages which differ in their directionality.

To date, only one study in the face perception literature has sought to examine the influence of directionality of script. Subjects in this study, by GILBERT and BAKAN [14], consisted of groups of right- and left-handed Americans, whose sole language was English, and right-handed Israelis, who were native speakers of Hebrew and had studied English in school. Their task was to decide which of two bisymmetric composite faces formed from left or right sides of a target face more closely resembled the target face. The results indicated a strong left field bias among the right-handed American subjects and a significant left field bias in the Israeli subjects, but for this group "the effect was ... weaker" ([14], p. 360). Unfortunately, since the authors treated stimuli, rather than subjects, as the random variable in their data analysis, it is not possible to compare the size of the leftward bias in the two groups. Thus, the present investigation represents the first attempt to examine the contribution of scanning effects vis-à-vis laterality effects on a nonverbal laterality task.

\section{METHOD}

\section{Subjects}

A total of 131 right-handed adults and 31 left-handers participated in the experiment. Handedness was assessed by self-ratings on a brief handedness inventory adapted from OLDFIELD [34]. Subjects were subdivided into six groups, including four right-handed groups and two left-handed ones. Group 1, the left-to-right readers, consisted of righthanded native users of Hindi and included 35 subjects ( 13 male, 22 female) with a mean age of 28.2 years. These subjects had studied English in school, but none of them knew the Urdu script or any other right-to-left script. Group 2, the bi-directional readers, contained 57 right-handed native users of Urdu (21 male, 36 female) with a mean age of 26.5 years. These subjects had studied the Hindi script in school, and some had also studied English in school. Group 3, the right-to-left readers, consisted of 17 right-handed native Arabic users (13 male, 4 female) with a mean age of 22.6 years who had been in the U.S. for a few months having come from Iraq and Saudi Arabia to pursue higher studies. This group's knowledge of English was very rudimentary. Group 4 contained 22 right-handed illiteratc Hindi/Urdu-speaking adults ( 8 malc, 14 femalc) with a mean age of 23.2 ycars. Group 5 consisted of $19 \mathrm{left}-$ handed readers of Hindi (13 males, 6 females) and Group 6 consisted of 12 left-handed Urdu readers ( 3 males, 9 females). Since the expression of left-handedness has until very recently been strongly discouraged in Indian society our sample of left-handers is somewhat younger than our right-handed group. The mean age was 12.8 years for Hindi left-handers and 14.3 years for Urdu left-handers. All groups, with the exception of the Arabic speakers, were tested in two north Indian cities. 
Stimuli

The stimulus set was exactly the same as that used by LEvY et al. [27], and contained 36 pairs of photos of each of 9 male posers ( 5 right- and 4 left-handers), consisting of asymmetric chimeric faces in which either the left or the right half of the person's face was smiling. See Levy et al. [27] for a detailed description of how the stimuli were prepared.

\section{Procedure}

Subjects were tested individually; the Indian sample was tested by native Hindi/Urdu speaking Indian research assistants and the Arab sample by an Iraqi research assistant.

Since in a study such as this there is a risk of experimenter bias, care was taken to insure that the examiners were blind as to the rationale underlying the experiment. Subjects were shown the stimulus faces in central vision in a freeviewing situation, using a test booklet format that LEVY et al. [27] found was sufficiently sensitive for eliciting asymmetries. Stimulus pairs in the booklet were counterbalanced such that the smile was on the left side in the top photo in half the cases and in the bottom photo in the remaining trials. For each stimulus pair, subjects were to decide which of the faces looked happier. The experimenter recorded whether the top or bottom face was chosen on each trial. Subjects had unlimited time to view the stimuli.

Responses were coded in terms of whether the smile in the face perceived as happier was to the viewer's right or left. Following LeVY et al. [27], a laterality measure was computed per subject from the total number of pairs in which a rightward response was chosen $\left(N_{\mathrm{R}}\right)$ minus the number of pairs in which a leftward response was chosen $\left(N_{\mathrm{L}}\right)$ divided by the total number of pairs $(N=36)$. Thus, a negative score would signal a leftward preference.

\section{RESULTS}

\section{Reliability}

To determine whether subjects' responses were internally consistent, a split-half reliability measure was computed per group, using the Pearson product moment correlation. The analysis revealed a significant coefficient of correlation between odd and even numbered trials for all of the right-handed groups with the exception of the illiterates; of the two lefthanded groups, only the Urdu subjects' responses yielded a significant corrclation coefficient. See Table 1 for a summary of the reliability scores.

Table 1. Odd even split-half reliability values on chimeric faces test

\begin{tabular}{ll}
\hline & \multicolumn{1}{c}{$r$} \\
\hline Right-handers & \\
Hindi & $0.707^{*}$ \\
Arab & $0.844^{*}$ \\
Urdu & $0.620^{*}$ \\
Illiterates & 0.015 \\
& \\
Left-handers & \\
Hindi & 0.181 \\
Urdu & $0.876^{*}$ \\
\hline
\end{tabular}

*Significant at $p<0.05$.

\section{Asymmetry values}

By way of comparison we may note that, for the 111 individually tested right-handers reported in LEVY et al. [27], the mean asymmetry score was -0.303 and for the 111 left handers the score was -0.134 . In the LEVINE and LEVY [25] study, the scores ranged from -0.181 to -0.331 across the various age ranges (Grade 1 to Grade 12) with the exception of the youngest group (kindergarteners) whose mean score of -0.092 differed significantly from the overall mean score of the older age groups. 
Table 2 provides a summary of the mean asymmetry values and standard errors of each group in the present study. The scores ranged from -0.286 in the Hindi right-handers to 0.072 in the Arab sample. Two separate analyses of variance were performed, one with the right-handers only, the other comparing left-handed Hindi and Urdu subjects with their right-handed counterparts.

1. Right-handers. Results of the analysis with the right-handed groups indicated no sex difference and a main effect of $\operatorname{Group}[F(3,130)=6.05, P<0.007]$. Single degree of freedom contrasts revealed that Hindi subjects were significantly different from all three other groups, $P<0.01$ (the $F$ values were $14.81,10.74$ and 7.37 for the comparisons of Hindi with Urdu, Arab, and illiterate subjects, respectively). There was no significant difference between Urdu and Arab subjects, nor between these two groups and the illiterates.

2. Right-vs left-handers. The analysis of variance comparing right- and left-handed Hindi and Urdu readers yielded no main effects of Group or Handedness but an interaction of the two $[F(1,119)=7.33 \quad P<0.007]$. Breakdown of the interaction revealed a significant difference between Hindi and Urdu right-handers and between Hindi right- and left-handers $(P<0.05)$. Urdu right- and left-handers did not differ; nor did Hindi and Urdu left-handers.

Table 2. Mean asymmetry scores on chimeric faces test

$$
(\mathrm{R}-\mathbf{L}) / 36
$$

\begin{tabular}{lrr}
\hline & Score & S.E.M. \\
\hline Right-handers & & \\
I Iindi & -0.286 & 0.066 \\
Urdu & 0.022 & 0.049 \\
Arab & 0.072 & 0.117 \\
Illiterate & -0.010 & 0.041 \\
Left-handers & & \\
Hindi & -0.035 & 0.047 \\
Urdu & -0.139 & 0.099 \\
\hline
\end{tabular}

\section{Percentage of subjects with a leftward bias}

By way of comparison, we may note that the majority of right-handed adults in previously published studies using this paradigm show a left-field preference: $75 \%$ in HeLLER and LEVY [18], 74\% in LEVY et al. [27]. The Levine and Levy study tested a broad age range; $72 \%$ of the subjects, excluding the youngest age group (5 years) showed a LVF preference.

Following LEvy et al. [27], we classified subjects as showing a left bias, a right bias or no bias using $P<0.10$ and $P<0.05$ levels of significance on a 1 -tailed test. Since the results did not differ across the two classifications, only those obtained for the $P<0.05$ level are reported below. We found that $68.6 \%$ of Hindi right-handed readers showed a significant asymmetry in either direction, as did $76.5 \%$ of the Arabic readers, $42.1 \%$ of Urdu right-handed readers, $33.3 \%$ of Urdu left-handers, $26.3 \%$ of Hindi left-handers and $18.2 \%$ of illiterates. A chi square analysis indicated that, of those in each group who showed a significant asymmetry, it was in the leftward direction for $83.3 \%$ of Hindi right-handers $(P=0.002$, with Yates correction, two-tailed), $54.2 \%$ of Urdu right-handers $(P=0.97$, with Yates correction, two- 
tailed), and only $30.8 \%$ of Arabic (right-handed) readers (Fisher's exact $P=0.27$, twotailed). Since the number of illiterates and Hindi and Urdu left-handers who showed any asymmetry at all were very small ( 5 or fewer subjects), the corresponding percentages for these groups $(50,40$ and $75 \%$, respectively) are probably not very meaningful. Chi square analyses further revealed a significantly greater leftward bias in right-handed Hindi than Urdu subjects, and between right-handed Hindi vs Arab subjects $(P<0.05)$. Table 3 summarizes the frequency data.

Table 3. Percentage of subjects who showed a significant $(P<0.05)$ left bias, right bias, or no bias.

\begin{tabular}{lrrr}
\hline & Left & Right & No bias \\
\hline Hindi right-handers $(N=35)$ & 57.14 & 11.43 & 31.43 \\
Urdu right-handers $(N-57)$ & 22.80 & 19.30 & 57.90 \\
Arabic right-handers $(N=17)$ & 23.53 & 52.94 & 23.53 \\
Illiterate right-handers $(N=22)$ & 9.09 & 9.09 & 81.82 \\
Hindi left-handers $(N=19)$ & 10.53 & 15.79 & 73.68 \\
Urdu left-handers $(N=12)$ & 25.0 & 8.33 & 66.67 \\
\hline
\end{tabular}

\section{DISCUSSION AND CONCLUSION}

The present findings reveal significant group differences in the size of the left visual field asymmetry observed on the free-vision facial affect perception test: the effect was strongest in right-handed readers of a left-to-right script (Hindi) and weakest in readers of a left-to-right script (Arabic). A frequency analysis further indicated that while the vast majority of Hindi readers showed a left field bias, Urdu readers showed no significant biases and Arabic readers, perhaps because of the smaller sample size, fell short of showing a significant right field bias. A frequency analysis comparing the incidence of a left field bias showed that Hindi right-handers were significantly more leftward oriented relative to Urdu and Arab righthanders (sèe Fig. 2).

Before discussing the results in terms of their bearing on the main issue, let us first examine some of the findings in more detail. According to a strict reading habit account the "pure" right-to-left group (Arab subjects) should have shown a larger rightward bias than the bidirectional group (Urdu subjects). While the numerical values of the $\Lambda$ rab group were in fact in the appropriate direction (0.072 vs 0.022 for the asymmetry scores, and $69.2 \%$ rightward vs $45.8 \%$ rightward for the frequency scores of Arab and Urdu subjects), group differences were not statistically significant. The lack of difference may reflect the small sample size of the Arab group, or may suggest that there is in fact no difference between solely right-to-left rcaders and native right-to-left readers who subsequently become bi-directional readers.

The lack of a significant difference between the mean asymmetry scores of the Arabic/Urdu readers and the illiterates is not surprising in view of the fact that the scores or the illiterates on this task were not statistically reliable (see Table 1), and over $80 \%$ of the illiterates showed no visual field bias on this task. The performance of the illiterates suggests that extralinguistic factors (e.g. lack of experience in test-taking) associated with illiteracy may lead to random responding. 

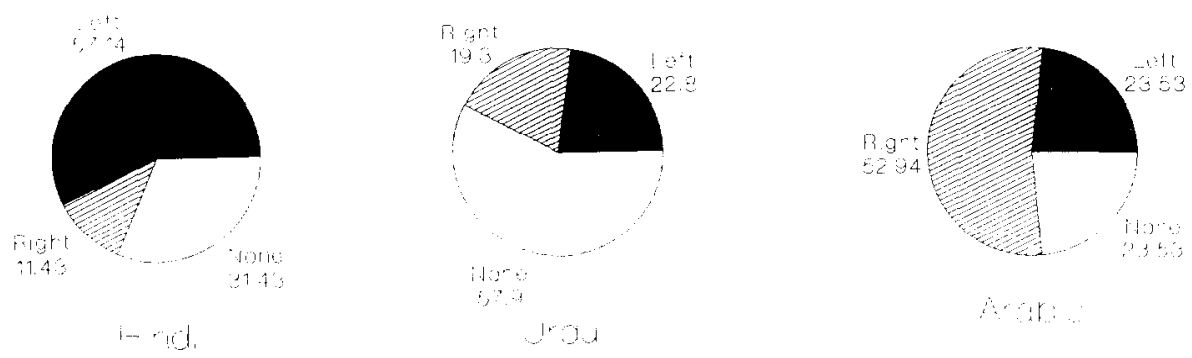

Fig. 2. Percentage of right-handed subjects who showed a significant, right side preference, left side preference, or no preference on the chimeric faces test.

The inclusion of left-handers in our sample allowed for a test of the "biological" explanation of the left field bias. While Hindi right-handers did show a stronger leftward bias as compared to Hindi left-handers, it is difficult to interpret this given that the scores of the Hindi left-handers were unreliable (see Table 1). Those of Urdu left- (and right-) handers were reliable and the lack of difference between these two groups would argue against a biological (i.e. handedness-based) account of the face perception bias. An alternative explanation is that the greater suppression of overt left-handedness in Muslim as compared to western cultures may lead to the inclusion of latent left-handers among the Urdu righthanded sample. If one accepts this possibility, though, it could be argued that individuals who do report left-hand preference in cultures in which this is not socially sanctioned might be particularly strongly left-handed, and thus, might show a different pattern of performance from forced right-handers, if the task is indeed a reflection of biological difference. Since no handedness difference was ohtained in the Urdu sample we may conclude that a reading habit explanation is at least in part a valid account.

Taken together, the present results suggest an influence of reading and writing experience on a task that has to date been regarded mainly if not exclusively as a measure of right hemisphere specialization for non-linguistic affect. It remains to be determined whether the reading habit effect reflects a difference in the spatial distribution of attention or a difference in mental scanning [17]. Studies of face scanning show no consistent asymmetries either in the direction of the initial gaze or in gaze duration (see [16] for further discussion).

Interestingly, the only group previously studied in the literature which did not show a robust left visual field preference for facial affect judgments consisted of pre-literate 5-yearolds [25]. Only $25 \%$ of the 20 subjects in this group showed a significant asymmetry at all. A reading-habit interpretation may at least in part account for this group's poor performance on the face judgment task in addition to fatigue or random responding on later trials, the explanation offered by LEVINE and LEVY [25]. The authors' attempt to explain the unique performance of the 5-year-olds' on an additional task (circle drawing) may not be entirely valid since this task has itself been shown to be influenced by reading habits [15].

Does there exist converging evidence to support a reading habit account of the present results? Behavioral studies comparing Hindi and Urdu/Arab readers have indicated opposite biases in attention to the left or right side of space in verbal as well as nonverbal tasks. On a figure drawing task, for example, most Hindi readers begin at the left side of an unlined page whereas most Urdu and Arab subjects begin at the right side of the page. Moreover, Hindi subjects tend to draw figures with intrinsic directionality (e.g. elephant, 
bicycle) in a leftward facing direction, whereas the majority of Urdu and Arab subjects tend to draw these figures facing right [39]. Thus, prior experience with a left-to-right vs a rightto-left writing system appears to direct attention to the left or right side of space, respectively, and influences the lateral orientation of nonlinguistic stimuli (see [31] for similar findings in the Hebrew literature).

While a reading habit explanation can account for the results in part, it is not the sole explanation, for the performance of the right-to-left groups was not a mirror-image of that of the left-to-right group. Thus, it is likely that reading habit interacts with cerebral lateralization-based differences to result, as in the present example, in an enhanced left field bias in the left-to-right group and an attenuated or absent left field bias in the right-to-left and bidirectional readers.

Interestingly, a similar interaction is not found when the task involves verbal processing, as was observed by VAID [38] in studies of Hindi vs Urdu readers, and by BELIN et al. [1] in Arabic vs French readers. This clearly indicates that, for verbal processing, a laterality effect overrides any scanning effect, at least when single words are used.

In conclusion, the present study indicates the need for caution in drawing inferences about hemispheric specialization from studies obtaining asymmetries in nonverbal performance that use only readers of a left-to-right language. Since the chimeric facial affect perception test appears to be susceptible to effects of directional biases arising from reading and writing experience, performance on this test cannot be interpreted solely in terms of hemispheric differences in the perception of facial affect. Inasmuch as reading habit influenced performance on the chimeric faces test, it is reasonable to rc-cxamine other face perception tasks, such as the composite faces test [16] or related tests in the nonverbal laterality literature with a view to determining the existence of reading-scan-related biases. For example, a left-sided bias has been reported for the location of self-generated images [22]. More recently, BoroD et al. [5] have reported that performance on this spatial visualization task corrclated significantly with a left field bias observed on the chimeric facial affect judgment task. Clearly, there may be some component of either task that taps hemispheric functional asymmetry; nevertheless, both tasks may also be influenced by reading habit. At the very least, the present research underscores the importance of using readers of left-toright and right-to-left scripts in investigations of cerebral lateralization of function, even for ostensibly nonlinguistic tasks, before inferences about hemispheric specialization of function can be drawn from observed perceptual or performance asymmetries.

Acknowledgements - This research was supported in part by an Indo-American Advanced Research fellowship awarded to J. Vaid by the Council for International Exchange of Scholars, and by a University Grants Commission fellowship in India awarded to M. Singh.

A preliminary report of this research was presented at the annual meeting of the Body for the Advancement of Brain, Behavior and Language Enterprises, Niagara Falls, Ontario, March 1986. Najah Abdel-Kadar assisted in collecting the Arab data and Abdur Rasheed assisted in collecting some of the Urdu and Hindi data. We thank Jerre Levy, Lauren J. Harris and Eta Schneiderman for comments on eariier drafts. Jorge Mendoza and Tom Battocletti assisted in the data analysis.

\section{REFERENCES}

1. Belin, C., Pionner, V., Perrier. D. and Larmanif, P. Tachistoscopic recognition of words in a right-to-left language: Arabic. J. clin. exp. Neuropsychol. 10, 1988.

2. Bertelson, P. Listening from left to right versus right to left. Perception 1, 161-165, 1972.

3. Bertelson, P. and Tisseyre, F. Set and lateral asymmetry in the perceived sequence of speech and nonspeech. In Atlention and Performance, Vol. 5, P. M. RABbiTt and S. Dornic (Editors), Academic Press, New York, 1972. 
4. BEST, C. Asymmetries in perception and production of infant emotional expressions in chimeric presentation. $J$. clin. exp. Neuropsychol. 8, 1987.

5. BOROD, J, Vingiano, W. and Cytryn, F. Neuropsychological factors associated with perceptual bias for emotional chimeric faces. Int. J. Neurosci. in press, 1989

6. BRYden, M. P. Left-right differences in tachistoscopic recognition: directional scanning or cerebral dominance? Percept. Motor Skills 23, 1127 1134, 1966.

7. BRYDEN, M. and LEY, R. Right-hemispheric involvement in the perception and expression of emotion in normal humans. In Neuropsychology of Human Emotion, K. M. Hellman and P. Satz (Editors), pp. 6 44. Guilford Press, New York, 1983.

8. Campbell, R. Asymmetries in interpreting and expressing a posed facial expression. Cortex 16, 473-481, 1978.

9. Campbell, R. The lateralization of emotion: a critical review. Int. J. Psychol. 17, 211-229, 1982.

10. Carlson, D. and Harris, L. Perception of happiness and sadness in free-viewing of chimeric faces. J. clin. exp. Neuropsychol. 7 (6), p. 636, 1985.

11. Carlson, D. and Harkis, L. Perception of positive and negative emotion in free-viewing of asymmetrical faces: sex and handedness effects. INS Bulletin, p. 9, 1984.

12. Corballis, M. Fresh fields and postures new: a discussion paper. Brain Cognit. 5, 240 252, 1986.

13. Freimuth, M. and WAPNER, S. The influence of lateral organization on the evaluation of paintings. $B r . J$. Psychol. 70, 211-218, 1979.

14. Gilbert, C. and BaKaN, P. Visual asymmetry in perception of faces: Neuropsychologia 11, 355--362, 1973.

15. Goodnow, J., Friedman, S., Bernbalm, M. and Lehman, E. Direction and sequence in copying: the effect of learning to write in English and Hebrew. J. Cross-cultural Psychol. 4, 263-282, 1973.

16. Gregl, D., Sackim, H., Sanciuez, E., Coiren, B. and Hougit, S. Perceiver bias in the processing of human faces: neuropsychological mechanisms. Cortex 24, 91-117, 1988.

17. Grover, R., W Alder, F. and Grover, M. Looking at faces: local and global aspects of scanning. In Theoretical and Applied Aspects of Fye Movement Research, A. Gaif and F. Johnson (Editors), pp 523.533 NorthHolland, Amsterdam, 1984.

18. Heller, W. and Levy, J. Perception and expression of emotion in right-handers and left-handers. Neuropsychologia 19, 267-272, 1981.

19. Hellige, J., BLOCh, M. and TAYloR, A. J. Multi-task investigation of individual differences in hemispheric asymmetry. J. exp. Psychol.: Hum. Percept. Perform. 14 (2), 176-187, 1988.

20. Heron, W. Perception as a function of retinal locus and attention. Am. J. Psychol, 70, 38-48, 1957.

21. JAeger, J., Borod. J. and Peselow, E. Depressed patients have a typical hemisphere bias in the perception of emotional chimeric faces. J. Abnorm. Psychol. 96 (4), 321-324, 1987.

22. Koff, E., Borod, J. and White, B. A left hemispace bias for visualizing emotional situations. Neuropsychologia 21 (3), 273-275, 1983.

23. Kugelmass, S. and Lieblich, A. The impact of learning to read on directionality in perception: a further cross-cultural analysis. Hum. Dev. 22, 406 415, 1979.

24. Lieblicit, $\Lambda$., Ninio, $\Lambda$. and Kugelmass, $S$. Developmental trends in directionality of drawings in Jewish and Arab Israeli children. J. Cross-cultural Psvchol. 6, 504 511, 1975

25. Levine. S. and Levy, J. Perceptual asymmetry for chimeric faces across the lifespan. Brain Cognit. 5, 291-306, 1986

26. LeVy, J. Lateral dominance and aesthetic preference. Neuropsychologia 14, 431-455, 1976.

27. LeVy, J., Heller, W., Banich, M. and Burton, L. Asymmetry of perception in free viewing of chimeric faces. Brain Cognit. 2, 404-419, 1983.

28. LeY, R. and STRauss, E. Hemispheric asymmetries in the perception of facial expressions by normals. In The Neuropsychology of Face Perception and Facial Expression, R. BRUYER (Editor), pp. 269-289. Lawerence Erlbaum, Hillsdale, New Jersey, 1986.

29. Lieblich, A., Ninio, A and Kuglemass, S. Developmental trends in directionality of drawing in Jewish and Arab Israeli children. J. Cross-cultural Psychol. 6, 504 511, 1975.

30. Mishikin, M., and Forgays, D. Word recognition as a function of retinal locus. J. exp. Psychol. 43, 43-48, 1952.

31. NachshoN, I. Directional preferences in perception of visual stimuli. Ini. J. Behav. Neurosci. 25, 161-174, 1985.

32. NaranG, G. C. Development and use of writing system across cultures: the case of Arabic-Persian Urdu orthographical model. J. South Asian and Middle-Eastern Studies 10 (2), 64 77, 1986.

33. Natale, M., Gur, R. E. and Gur, R. C. Hemispheric asymmetries in processing facial expressions. Neuropsychologia 21, 555-566, 1986.

34. OldFiEld, R. C. The assessment and analysis of handedness: The Edinburgh Inventory. Neuropsychologia 9 , $97-113,1971$.

35. Orbach, J. Differential recognition of Hebrew and English words in right and left visual fields as a function of cerebral dominance and reading habits. Neuropsychologia 5, 127-134, 1967.

36. SACKeIm, H. and Gur, R. C. Facial asymmetry and the communication of emotion. In Social Psychophysiology: A Sourcehook, J. Cacioppo and R. PETTY (Editors). Guilford, New York, 1983. 
37. Tramer, O., Butler, B. and Mewhort, D. Evidence for scanning with unilateral presentation of letters. Brain Lang. 25, 1-18, 1985.

38. VAID, J. Asymmetries in tachistoscopic word recognition: scanning effects re-examined. Int. J. Neurosci. 42, $253-258,1988$.

39. VAID, J. Effect of reading and writing direction on nonlinguistic perception and performance: Hindi and Urdu data. Scripts and Reading, I. TAYLOR and D. OLSON (Editors). Kluwer Academic, Dordrecht, in press, 1989. 